\title{
Global partnerships are critical to advance the control of Neglected Zoonotic Diseases: The case of the Global Alliance for Rabies Control
}

Louis $\mathrm{H} \mathrm{Nel}^{1,2}$, Louise H Taylor* ${ }^{* 1,3}$, Deepashree Balaram ${ }^{1}$ and Kim AS Doyle ${ }^{1}$

*Corresponding Author, email: Louise.Taylor@rabiesalliance.org, Phone: +1 4122412480

${ }^{1}$ Global Alliance for Rabies Control, 529 Humboldt Street, Suite 1, Manhattan, Kansas 66502, USA

${ }^{2}$ Department of Microbiology and Plant Pathology, Faculty of Natural and Agricultural Sciences, University of Pretoria, Pretoria 0001, South Africa.

${ }^{3}$ Boyd Orr Centre for Population and Ecosystem Health, Institute for Biodiversity, Animal Health \& Comparative Medicine, University of Glasgow, Glasgow, United Kingdom.

\section{KEYWORDS}

Rabies; Rabies control; Neglected Zoonotic Disease; Health Policy 


\section{ABSTRACT}

There is a need for innovation to improve control of all Neglected Zoonotic Diseases (NZDs). The Global Alliance for Rabies Control was formed to prevent human deaths from rabies and relieve the burden of rabies in other animal populations, especially dogs. It aims to identify reasons for the neglect of rabies in developing countries and to develop strategies to improve rabies control. Through initiatives such as World Rabies Day and the Partners for Rabies Prevention, progress has been made towards increased awareness of the burden of rabies transmitted by dogs at scales from local to international. An evidence base of the feasibility of canine rabies elimination has been built up and now easier access to information and tools enables countries to design and implement rabies elimination strategies in a logical way, utilizing the structures of regional networks for rabies control. The body of evidence has built consensus amongst international stakeholders in rabies control and is now being used to encourage international policy change, attract investment and increase delivery of effective rabies control programmes in canine rabies endemic countries.

\section{INTRODUCTION}

Since zoonoses are by definition animal diseases that are transmissible to humans and often have significant impact on public health, these diseases require an intersectoral approach to effectively control them. Because of this complexity and given that many zoonoses primarily impact poor rural communities of the developing world, they tend to be become neglected. Rabies is an excellent albeit unfortunate example of such a neglected zoonotic disease (NZD). This disease is today recognized as one of eight NZDs by the World Health Organization (WHO)(WHO, 2015a), in recognition of the lack of progress in many countries all around the world with regard to the control of canine rabies. This lack of prioritization results 
in underinvestment in rabies control activities, which could be addressed by building a case for the costs and benefits of appropriate investments (Shwiff et al., 2013).

Over the past 2 decades, it has gradually become apparent that new and innovative approaches are required to turn around the chronic neglect of NTDs (WHO, 2012). One innovative concept to materialise in this process has been the recognition of the potential of Public Private Partnerships (PPPs). Such PPPs could meaningfully address the neglect of some diseases that should demand much higher priority, given their impact on veterinary and public health (Buse and Walt, 2000, WHO, 2002, Widdus, 2005). Significant progress has been demonstrated by PPPs geared towards addressing other global public health issues, including the Global Alliance to Eliminate Lymphatic Filariasis (Addiss and Global Alliance to Eliminate Lymphatic Filariasis, 2010), the Roll Back Malaria Partnership (Roll Back Malaria Partnership, 2010), GAVI (Lob-Levyt, 2011) and the London Declaration group (Gulland, 2012).

The Global Alliance for Rabies Control (GARC), a non-governmental organisation established in 2007, set up the first PPP in the NZD field. The strategy and achievements of GARC with its partners are the subjects of this paper.

While rabies has evolved to infect all mammals, particularly carnivores, the domestic dog populations of the developing world are the most significant reservoir and vector for human rabies today. However, dog rabies is preventable. Vaccines to eliminate the disease in dogs have been available for decades and have been successfully applied in Western Europe, North America and more recently Latin America. Similarly, highly efficacious vaccines for use in humans have been available for a long time. Apart from the possibility of pre-exposure vaccination, the utility of post-exposure treatment to prevent the onset of disease even after exposure to a rabid animal is well established ( $\mathrm{Nel}, 2013$ and references therein). While rabies was an established scourge in parts of Europe and the Americas by the $17^{\text {th }}$ and $18^{\text {th }}$ centuries, the dramatic spread of dog rabies in Africa and Asia occurred only during the 1900s (Nel and Markotter, 2007, Nel and Rupprecht, 2007). In Africa, several hundred million people are currently estimated to be at risk of endemic dog rabies, with more than 20,000 human deaths annually. In Asia, 
current estimates indicate as many as 35,000 human deaths annually (Hampson et al., 2015). Given the proven measures of control and preventability, the burden of rabies in the developing world is a major indictment of veterinary and public health strategies, practices and priorities and demonstrates that Universal Health Coverage remains a distant goal.

Given the above perspectives, the non-profit Global Alliance for Rabies Control (GARC), with its formation in 2007 , committed to the development of strategies to prevent human deaths from rabies and relieve the burden of rabies in other animals, especially dogs. From the outset GARC was built not only around the need for an integrated One Health intersectoral partnership approach, but also the realization that such partnerships would need to be functional on all the different levels of disease control, viz. the global, regional, national and community levels.

In the first phase of the programmatic development of GARC, the organisation took a lead on raising awareness of rabies and its neglect. This goal was achieved through the creation and coordination of an annual World Rabies Day (WRD) and the Partners for Rabies Prevention (PRP) group, which united all the major international stakeholders involved in rabies control.

\section{RAISING AWARENESS AND ENHANCING INTERSECTORAL COOPERATION}

\subsection{World Rabies Day}

GARC's first major partnership initiative, with among others the World Health Organization (WHO), the World Organisation for Animal Health (OIE), the UN Food and Agriculture Organization (FAO), the US Centers for Disease Control and Prevention (CDC), and the Pan-American Health Organisation (PAHO), was the creation of an annual World Rabies Day (WRD, www.rabiesalliance.org/world-rabies-day/) (Briggs and Hanlon, 2007). The endorsement by the international health organizations and encouragement of 
their member states to participate in World Rabies Day (OIE, 2011), have also enabled many governments to use the opportunity of WRD to launch or support improvements in rabies control. By focusing on partnerships, it was possible to maximise the limited resources available and to provide geographic reach through existing physical and virtual networks.

The inaugural World Rabies Day in 2007 saw events in 74 countries and over the past 7 years, more than 150 countries have participated, with educational outreach to millions of people worldwide. With the support of GARC there was, on the occasion of WRD 2013, a joint call from FAO,OIE and WHO for the support of global canine rabies elimination (FAO, 2013), which was a major milestone in the collaboration between international agencies. In 2014, 260 WRD events were registered with GARC and displayed on its website. GARC also coordinates specific partnership initiatives to increase its reach. For example, in 2014, a Global One Health competition for teams of medical and veterinary students to execute WRD initiatives yielded 28 submissions from 17 countries. This project was implemented in partnership with the International Veterinary Students' Association and International Federation of Medical Students' Associations.

The principal objective and achievement of WRD was the generation of widespread awareness of key rabies prevention messages about the fatality of rabies, the preventability of rabies, potential for elimination of rabies and the unfortunate state of neglect of this disease. It is therefore also no coincidence that WRD falls on 28 September, the anniversary of Louis Pasteur's death. Part of its success lies in the concept of WRD being fundamentally inclusive, creating opportunities for a vast variety of actions and messages by participants ranging from global intergovernmental organizations to small NGOs, and reaching different target groups and individuals around the world including teachers, children, academics, and health professionals.

Education messages and models have been developed and promoted as part of the key rabies prevention activities. These have ranged from poster outreach in Africa, supported by FAO distribution channels; to support for the integration of rabies education into school curriculums, piloted by the government of the 
Philippines. In the most recent development, the WRD partnership framework will support the dissemination of the GARC Rabies Educator Certificate. This is a free web-based course for individuals such as community educators and health workers who would like to learn about rabies and how to teach others to prevent rabies and reduce deaths in their communities (GARC, 2015a).

\subsection{Partners for Rabies Prevention (PRP)}

Building on the initial success of WRD, GARC's next partnership initiative was to gather major stakeholders in the field of rabies prevention and control to share ideas and work collaboratively towards identifying and overcoming the main obstacles to the effective control and eventual elimination of dog rabies. To do so was a major challenge, considering the plethora of stakeholders ranging from intergovernmental organizations, international institutions and governmental agencies to nongovernmental organisation, academia and industry. GARC approached this by creating a new informal network, the Partners for Rabies Prevention (PRP), in 2008, to provide a unified platform that would allow for collective strategic thinking and planning around the diverse skills, experience, and capabilities of the global spectrum of partners.

The PRP was modelled on the Partners for Parasite Control (PPC), which is composed of agencies of the United Nations, WHO Member States, research institutes and a multitude of NGOs (WHO, 2001). The PRP functions as a technical arm for GARC while also uniting and focusing the global rabies community on the common objective of dog rabies elimination (Lembo et al., 2011, Nel, 2013). GARC, serving as the Secretariat and convenor of the PRP, arranges an annual meeting and sets the meeting agenda. The organizations and individuals involved in each annual meeting reflect the agenda to be discussed, and meetings have included key representatives from international health organizations (FAO, OIE, WHO), academic institutions, foundations, NGOs, rabies vaccine manufacturers, WHO collaborating centres, OIE reference centres, rabies expert networks and governments of rabies endemic countries. Many partners within the PRP have a long history of facilitating country efforts to control rabies, and their combined 
expertise in rabies control and other relevant fields has contributed to the creative and solutionorientated environment that has characterised PRP meetings to date. To accommodate the range of activities, the partnership agreements vary in scale and focus from broad based umbrella agreements focusing on global rabies elimination to technical agreements with sub-national authorities focusing on operational research. Formal collaborative agreements with the multilateral organizations (FAO, OIE, WHO) support global coordination and elimination plans. The addition of representatives from foundations (UBS Optimus Foundation, Bill and Melinda Gates Foundation) and rabies vaccine manufacturers (Sanofi Pasteur, Novartis, Merck Animal Health, Merial) allowed the PRP to benefit from global health insights, market data and corporate planning. Eight annual GARC/PRP meetings have been hosted to date.

At the community level, to increase access to information for professionals dealing with rabies in some way , a newsletter was created as far back as 2006 (GARC, 2015b), with reader contributions invited to increase community buy-in and expand the audience reach of local rabies efforts. Through this communication mechanism over 7,500 individuals interested in rabies control, many of whom work in rabies endemic countries, are currently kept up to date on developments and recent research in rabies control.

\section{BUILDING THE EVIDENCE BASE FOR CHANGE}

An in-depth analysis by the PRP in 2009 sought to identify gaps in the rabies landscape and global rabies policy that were preventing progress in rabies control. Some of the key barriers identified were the lack of data on the human and economic burden of the disease, poor coordination of national and international efforts, a lack of awareness concerning the most appropriate and effective rabies control mechanisms, limited availability of and capacity to deliver vaccines, too few successful demonstration projects and very limited engagement of key policy makers in endemic countries (Lembo et al., 2011). This 
analysis informed the work of the PRP going forward was used to establish specific applied research priorities and advocacy efforts (Figure 1), and sought to address the traditional research-policy disconnect (Kakkar et al., 2012, Yin et al., 2013). The resulting applied research plans addressed communication and education efforts, the challenges of limited intersectoral collaboration, and technical research questions (e.g. new laboratory surveillance tools). Various members of the PRP were involved in the execution of the prioritised research needs with support and coordination by GARC. Some research gaps could not be addressed by the initial PRP group and GARC created other bilateral and multilateral collaborations and coalitions to focus on these gaps. Ventures were set up for specific research, field models, advocacy and communications projects, and the partners included organisations operating at the national and sub-national level in the fields of human and animal health and welfare. Overall, an important element of this approach was the understanding that intersectoral stakeholder partnerships ranging from global to municipal levels are necessary to successfully tackle the challenges of rabies prevention. 
Figure 1: Rabies landscape analysis used to set research priorities by the PRP group

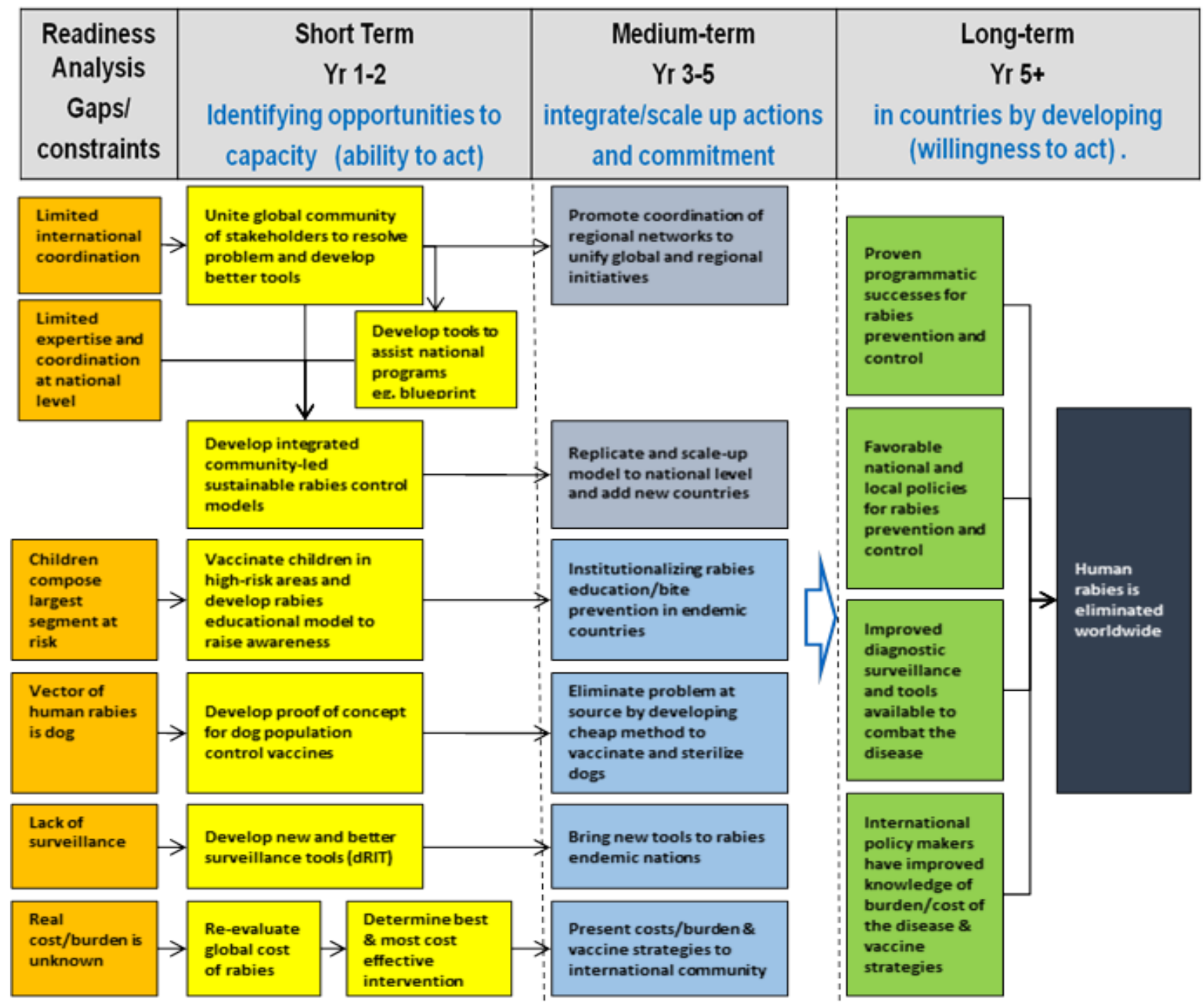

\subsection{The Blueprint for Rabies Prevention and Control}

An illustration of the success of the PRP platform was the development and launch of the Blueprint for Rabies Prevention and Control (www.rabiesblueprint.com). This blueprint was developed to meaningfully assist potential or existing national and international rabies interventions, and is essentially an operational toolkit for rabies elimination built on the principles of the One Health concept and presented 
in a user-friendly format (Partners for Rabies Prevention, 2010, Lembo and Partners for Rabies Prevention, 2012). To date there are three components to the rabies blueprint: Canine Rabies Control; Fox Rabies Control; and Rabies Surveillance (applicable to any host species) which are freely available online and regularly updated. It is envisaged that this tool will be expanded to become a central access point from which users can access all the tools, standards and information relevant to effectively control rabies in their country.

The Stepwise Approach towards Rabies Elimination (SARE) was added to the Canine Rabies Blueprint at the end of 2014. The SARE is a planning and self-assessment tool that countries may use to develop activities and measure progress towards a national programme and strategy for sustainable rabies prevention, control and eventually elimination, focusing on the prevention of dog-transmitted human rabies. Each stage of the SARE is characterized by a set of objectives to be reached and builds on the previous successes achieved (e.g. experience of a pilot area is scaled up to national level)(Partners for Rabies Prevention, 2014).

\subsection{Demonstration rabies control projects}

Building on initiatives to raise awareness and enhance multisectoral cooperation, the next priority was to generate and promote evidence that demonstrates the potential to successfully control dog rabies to the extent that elimination of the disease becomes a realistic and credible objective. In doing so, proof of concept was identified as an important component of persuading policy makers of the value of canine rabies control. GARC supported local and government partners, to deliver evidence of successful control on the island of Bohol in the Philippines, with an intersectoral approach based on canine and human vaccination, also involving the legislative and education sectors, with the crucial incorporation of active community engagement (Lapiz et al., 2012). This approach is currently being followed to bring rabies control to other regions of the Philippines and Indonesia. 
PRP members were actively engaged in the choice of sites for the large-scale canine rabies control programmes supported by the Bill and Melinda Gates Foundation, coordinated by the WHO. These largescale intersectoral rabies projects in South Africa, Tanzania and the Philippines have demonstrated that mass canine vaccination as a means of controlling rabies is possible and can be successfully catalysed with appropriate support and with close cooperation of the veterinary health and public health sectors (WHO and Bill \& Melinda Gates Foundation, 2015, Shwiff et al., 2014, Miranda et al., 2015, Hatch B et al., in review, Nel et al., 2009).

\subsection{Applied Research}

A number of applied research opportunities specifically related to an effort to build the evidence base for change. The first was to utilize all of the cooperative networks created in the PRP to reassess the burden of canine rabies. The purpose of this work was to calculate, for the first time, the true global impact of rabies and to provide estimates for the impact in each country (Hampson et al., 2015). The second study addressed critical questions of the quality of global surveillance for human rabies, including the notifiability status of the disease in the different nations of the world (Taylor et al., 2015).

Progress in the rabies elimination showcase projects then allowed another research opportunity, an exploration of the health economic aspects and potential benefits of successful rabies control. A health economics working subgroup of the PRP was formed, to explore how to best assess the costs of rabies to the economy of a country, and the resultant economic benefits that canine rabies elimination might realise (Shwiff et al., 2013). Such analyses have shown how investment in canine rabies prevention would realise future economic benefits of reduced social burden, expenditure on post exposure vaccination of bite victims, and lost income associated with rabies exposures. Further detailed economic assessments of canine rabies vaccination projects have also provided data on the costs of such programmes (Shwiff et al., 2014). Once such data are available for a variety of settings across the world, it will be possible to estimate the potential costs and benefits of elimination on a larger scale. 
Through activities intended to spread rabies prevention information GARC has also united a global community to build advocacy for increased resources to control rabies. Pilot projects in Asia and Africa have demonstrated that sustained canine vaccination efforts work, not just in theory, but also in practice in regions traditionally least capable of reducing the burden of rabies. New evidence, particularly from health economic analyses and the canine rabies burden reassessment, is available on which to base policy and practical control decisions (Anderson and Shwiff, 2013, Shwiff et al., 2013, Shwiff et al., 2014, Hampson et al., 2015). The continual evolution of the rabies blueprint, including the addition of the SARE, has been instrumental in creating a system for gradual and tangible progress in rabies control across the world. Together these strands have formed a solid foundation on which to build a united expert opinion on the way forward for canine rabies control, confirmed in recent WHO and OIE guidance (WHO, 2013, OIE, 2014) and highlighted by tripartite statements on the fact that canine rabies elimination is a feasible goal (FAO, 2013).

\section{TRANSLATING EVIDENCE INTO POLICY CHANGE}

With the evidence mounting about not only the desirability of canine rabies elimination at a global scale, but also its feasibility (Lembo et al., 2010, Lankester et al., 2014, Cleaveland et al., 2014), GARC has moved to the third phase of its activities as it uses the proven evidence base and international coordination mechanisms to support policy change and advocate for national, regional and global elimination of rabies. It is now using all the evidence and tools generated to date to support countries as they move forward in their rabies control efforts.

To complement the PRP at the regional level, GARC is working with key stakeholders to strengthen regional platforms and foster exchanges between different countries and advocate for and improve national rabies control plans. This partnership-led approach has resulted in the replacement of the two existing networks in Africa, the African Rabies Expert Bureau and the Southern and Eastern African Rabies 
Group, with a Pan-African Rabies Control Network (PARACON). A collaborative agreement with Fondation Merieux has been set up to support the coordination of the Middle East and Eastern Europe Rabies Expert Bureau (MEEREB). In Asia, GARC is supporting the working groups on rabies within the Association of Southeast Asian Nations (ASEAN) and South Asian Association for Regional Cooperation (SAARC) as they develop their regional strategies. The Asian, African and the Middle Eastern delivery platforms will support national and regional control efforts by providing the tools and evidence base developed by GARC and PRP partners. The Rabies Blueprint and the Stepwise Approach towards Rabies Elimination will be advocated at regional rabies control workshops and meetings in Africa and Asia where dog rabies control strategies are critically needed. This approach has already been applied in the development of the National Rabies Control Programme for Kenya (Ministry of Health and Ministry of Agriculture Livestock and Fisheries, 2014) and the intention is to help all countries in these regional networks to follow suit.

\section{DISCUSSION}

The approach and strategy of GARC with its partners as outlined here, are broadly applicable to all neglected zoonotic diseases, viz. reviewing the evidence, finding the knowledge gaps, demonstrating success in case studies, conducting applied research to increase the evidence base, promoting tools to support country efforts and then using this information to build the case for investment in control efforts. The lack of intersectoral collaboration and partnerships amongst stakeholders negatively affects the control of all NZDs. Although improving the control of different NZDs may require different specific strategies, this process of identifying the barriers, the possible solutions, and building the partnerships required to enact change are applicable to any NZD.

The landscape is changing, with the emergence of regional and sub-regional rabies elimination roadmaps, which are at different stages of advancement and capacity, and there is some overlap and competition between networks. Although historically, scientific networks did not communicate much with the policy 
and strategy networks, this is changing and there are more open exchanges now, as understanding increases that an integrated 'model to policy' approach is needed to address the challenge. The medical and veterinary networks have also tended to work without collaboration, even though a 'One Health' intersectoral approach is needed to effectively tackle a zoonotic disease such as rabies. Collaboration is increasing between these sectors as well, along with other stakeholders such as educators and public health workers.

Many steps towards international canine rabies elimination are currently being taken. Besides national strategies developed for individual countries, several regional strategies for the elimination of canine rabies already exist or are under development. These are for the member countries of the Pan American Health Organization, ASEAN Plus Three (China, Japan and Korea), WHO South East Asia and Western Pacific Regional Offices.

A reinvigorated tripartite agreement has resulted in better coordination between the OIE, FAO and WHO to tackle the disease at the global level (FAO/OIE/WHO, 2010). The GARC Partners for Rabies Prevention group has brought major stakeholders together to share information, coordinate approaches and review and address global gaps in research, communication and policy.

The work conducted over the last 8 years by GARC and its partners have brought about many positive changes. There is now consensus on how canine rabies can be eliminated and global momentum towards this objective has begun. However, more involvement is needed. Much of this work remains at the proof of concept stage, and real progress on the ground in Africa and Asia is limited to a few countries where pilot projects and government programmes are in place.

Along the way challenges have been faced and lessons learned. Despite efforts, some specific goals such as the development of injectable dog sterilization techniques have not materialized. It is challenging for an NGO to raise funds that are not directed to specific project deliverables, and funding GARC's advocacy work and support to countries has been a challenge. For this reason, GARC is frequently unable to respond to country requests for material support even where the need is great. Now, as rabies starts to 
move up the global health agenda, GARC must commit to its key strengths and roles that are able to fill the gaps relative to the mandate of other global partners. High on this agenda would be raising awareness amongst the public and policy makers and a global advocacy campaign to end human deaths due to canine rabies.

GARC will maintain its emphasis on bringing partners together to focus on the key global issues in rabies control and will continue to invest in regional rabies control networks. These are platforms for the development and launching of national rabies control plans, and they support trans-boundary collaboration. Comparatively, countries would be able to continually measure their progress and plan the sequence of key future steps towards the execution of national rabies control and elimination plans. There remains a need for GARC to promote new evidence and tools that enable countries to plan and implement programmes based on existing knowledge, guidance and standards, assess the impact of financial investment in rabies control for their specific situation, develop advocacy to increase investment in rabies control, and self-assess their progress towards rabies elimination.

Without active support from the international health and development community, especially major donors, there is a risk that neither the existing national success stories nor the growing evidence base will be sufficient to catalyse rapid action elsewhere in Asia and Africa. Long term funding is needed from international bodies to assist rabies endemic countries to set up sustainable rabies control programmes. Led by the tripartite group (FAO/OIE/WHO) and GARC, a 'Rationale for Investment' document has been created, aimed at drawing in future resources and donors to support global canine rabies elimination (WHO, 2015b). Some gaps still remain, most notably in the development of a safe, ethical, effective and cost-effective product for dog population management that remains firmly in the development stage at this time.

The global neglected disease agenda is moving away from individual disease control strategies towards a more integrated disease control approach tackling several diseases at once (WHO, 2015a, Gulland, 2012). There is a need to investigate how to build integrated disease control programs including other neglected 
public health problems that affect rabies endemic regions of Africa and Asia and affect the same populations of marginalized society.

In disease control, the importance of targets and timelines is well recognised and the global rabies community has been discussing a target for a rabies-free world. To set the target, an agreed global strategy is required and would include incremental steps and mechanisms to measure progress towards the elimination target. This would require each of the partners to contribute according to their strengths, and coordination between partners on all levels will need to be maximized. In order to reach a rabies-free world, the strategy also needs mechanisms for measured impact in each individual dog rabies endemic nation, stressing the importance of functional and effective regional rabies control networks.

A number of regions have already set targets for canine-mediated rabies elimination - the Americas by 2015, ASEAN Plus 3 by 2020, WHO South East Asia Regional Office by 2020. Given the current state of progress in regions across the world, and the fact that all the tools are available to end this disease, global elimination of human deaths due to canine-mediated rabies is a realistic and achievable goal. The main stumbling blocks towards implementing these steps are a lack of political will and funding at the global, regional and national levels, leading to major national gaps in knowledge, infrastructure, access to vaccines, public awareness and sustained diagnostic and surveillance mechanisms. In this regard the role of GARC, in the context of a PPP, will be to help strengthen the case for future investments, by the countries themselves, international donors, and also new partners. To this end, during 2015 GARC will seek collaboration with rabies and broader public health stakeholders to launch a communications and advocacy campaign to end human deaths due to canine rabies.

On $6^{\text {th }}$ July 1885 , Louis Pasteur successfully tested his rabies vaccine on a 9-year-old boy, Joseph Meister, who had been attacked by a rabid dog. The boy survived and avoided contracting rabies. 130 years later, no one should die from a rabies exposure. It is time to set a global elimination timetable and campaign to end canine-mediated rabies. 


\section{ACKNOWLEDGEMENTS}

The authors are supported by funding to the Global Alliance for Rabies Control from the UBS Optimus Foundation.

\section{REFERENCES}

Addiss, D. \& Global Alliance to Eliminate Lymphatic Filariasis 2010. The 6th Meeting of the Global Alliance to Eliminate Lymphatic Filariasis: A half-time review of lymphatic filariasis elimination and its integration with the control of other neglected tropical diseases. Parasit Vectors, 3, 100.DOI:10.1186/1756-3305-3-100.

Anderson, A. \& Shwiff, S. A. 2013. The Cost of Canine Rabies on Four Continents. Transbound Emerg Dis.DOI:10.1111/tbed.12168.

Briggs, D. \& Hanlon, C. A. 2007. World Rabies Day: focusing attention on a neglected disease. Vet Rec, 161, 288-9.

Buse, K. \& Walt, G. 2000. Global public-private partnerships: Part I--A new development in health? Bull World Health Organ, 78, 549-61.

Cleaveland, S., et al. 2014. Rabies control and elimination: a test case for One Health. Vet Rec, 175, 18893.DOI:10.1136/vr.g4996.

FAO. 2013. FAO, OIE and WHO unite for World Rabies Day to call for elimination of disease [Online]. Available at: http://www.fao.org/news/story/en/item/198087/icode/ (Accessed on October 11th 2015).

FAO/OIE/WHO 2010. The FAO-OIE-WHO Collaboration. Sharing responsibilities and coordinating global activities to address health risks at the animal-human-ecosystems interfaces. A Tripartite Concept Note.

GARC. 2015a. GARC Education Programs [Online]. Available at: https://education.rabiesalliance.org/login/index.php (Accessed on October 13th 2015).

GARC. 2015b. Newsletter archives [Online]. Available at: http://rabiesalliance.org/media/newsletterarchive/ (Accessed on October 13th 2015).

Gulland, A. 2012. Governments and drug companies pledge to eliminate 10 neglected tropical diseases by 2020. BMJ, 344, e773.DOI:10.1136/bmj.e773.

Hampson, K., et al. 2015. Estimating the global burden of endemic canine rabies. PLoS Negl Trop Dis, 9, e0003709.DOI:10.1371/journal.pntd.0003709.

Hatch B, et al. in review. Towards canine rabies elimination in Dar es Salaam, Tanzania: Assessment of health economic data. Transboundary and Emerging Diseases.

Kakkar, M., et al. 2012. Moving from rabies research to rabies control: lessons from India. PLoS Negl Trop Dis, 6, e1748.DOI:10.1371/journal.pntd.0001748.

Lankester, F., et al. 2014. Infectious Disease. Implementing Pasteur's vision for rabies elimination. Science, 345, 1562-4.DOI:10.1126/science.1256306.

Lapiz, S., et al. 2012. Implementation of an intersectoral program to eliminate human and canine rabies: The Bohol Rabies Prevention and Elimination Project. PLOS Neglected Tropical Diseases. , 6, e1891.DOI:10.1371/journal.pntd.0001891.

Lembo, T., et al. 2011. Renewed global partnerships and redesigned roadmaps for rabies prevention and control. Vet Med Int, 2011, 923149.DOI:10.4061/2011/923149. 
Lembo, T., et al. 2010. The feasibility of canine rabies elimination in Africa: dispelling doubts with data. PLoS Negl Trop Dis, 4, e626.DOI:10.1371/journal.pntd.0000626.

Lembo, T. \& Partners for Rabies Prevention 2012. The Blueprint for Rabies Prevention and Control: A Novel Operational Toolkit for Rabies Elimination. PLoS Negl Trop Dis, 6, e1388.DOI:10.1371/journal.pntd.0001388.

Lob-Levyt, J. 2011. Contribution of the GAVI Alliance to improving health and reducing poverty. Philos Trans R Soc Lond B Biol Sci, 366, 2743-7.DOI:10.1098/rstb.2011.0040.

Ministry of Health and Ministry of Agriculture Livestock and Fisheries. 2014. Strategic Plan for the Elimination of Human Rabies in Kenya 2014 - 2030. [Online]. Nairobi. Available at: http://zdukenya.org/programme-1/ (Accessed on October 13th 2015).

Miranda, L. M., et al. 2015. Towards Canine Rabies Elimination in Cebu, Philippines: Assessment of Health Economic Data. Transbound Emerg Dis.DOI:10.1111/tbed.12350.

Nel, L., et al. 2009. Rabies Control Program in South Africa. Microbe, 4, 61-65.

Nel, L. \& Markotter, W. 2007. Lyssaviruses. Critical Reviews in Microbiology, 33, 301-324.

$\mathrm{Nel}$, L. H. 2013. Factors Impacting the Control of Rabies. Microbiol. Spectrum, 1, OH-00062012.DOI:10.1128/microbiolspec.OH-0006-2012.

Nel, L. H. \& Rupprecht, C. E. 2007. Emergence of Lyssaviruses in the old world: The case of Africa. Curr Top Microbiol Immunol, 315, 161-193.

OIE. 2011. Recommendations from the OIE Global Conference on Rabies Control, 7-9 September 2011, Incheon-Seoul (Republic of Korea) [Online]. Available at:

http://www.oie.int/fileadmin/Home/eng/Conferences Events/docs/pdf/recommendations/A Re commendation Global\%20Rabies\%20Conference\%20Seoul final.pdf (Accessed on October 13th 2015).

OIE. 2014. Rabies Portal [Online]. Available at: http://www.oie.int/animal-health-in-the-world/rabiesportal/ (Accessed on October 13th 2015).

Partners for Rabies Prevention. 2010. Blueprint for Rabies Prevention and Control [Online]. Available at: http://www.rabiesblueprint.org (Accessed on October 13th 2015).

Partners for Rabies Prevention. 2014. The Stepwise Approach towards Rabies Elimination [Online]. Available at: http://caninerabiesblueprint.org/A-stepwise-approach-to-planning (Accessed on October 13th 2015).

Roll Back Malaria Partnership 2010. Saving Lives with Malaria Control: Counting Down to the Millennium Development Goals. Progress and Impact Series. World Health Organization.

Shwiff, S., et al. 2013. Potential economic benefits of eliminating canine rabies. Antiviral Res, 98, 3526.DOI:10.1016/j.antiviral.2013.03.004.

Shwiff, S. A., et al. 2014. Towards Canine Rabies Elimination in KwaZulu-Natal, South Africa: Assessment of Health Economic Data. Transbound Emerg Dis.DOI:10.1111/tbed.12283.

Taylor, L. H., et al. 2015. Surveillance of Human Rabies by National Authorities - A Global Survey. Zoonoses Public Health.DOI:10.1111/zph.12183.

WHO. 2001. Partnership: The Partners for Parasite Control [Online]. Available at: http://www.who.int/intestinal worms/partnership/en/ (Accessed on October 13th 2015).

WHO. 2002. Dr Gro Harlem Bruntland's Address to the Fifty-fifth World Health Assembly [Online]. Available at: http://www.who.int/directorgeneral/speeches/2002/english/20020513 addresstothe55WHA.html (Accessed on October 13th 2015).

WHO 2012. Accelerating work to overcome the global impact of neglected tropical diseases. A roadmap for implementation. Geneva: WHO.

WHO 2013. WHO Expert Consultation on Rabies, Second report. WHO Technical Report Series 982. Geneva: World Health Organization.

WHO 2015a. Investing to overcome the global impact of neglected tropical diseases: third WHO report on neglected diseases 2015. Geneva: World Health Organization. 
WHO 2015b. Rabies: Rationale for investing in the global elimination of dog-mediated human rabies. World Health Organization.

WHO \& Bill \& Melinda Gates Foundation 2015. Report of the sixth meeting of the International Coordinating Group of the World Health Organization and the Bill \& Melinda Gates Foundation project on eliminating human and dog rabies Geneva: WHO.

Widdus, R. 2005. Public-private partnerships: an overview. Trans R Soc Trop Med Hyg, 99 Suppl 1, S18.DOI:10.1016/j.trstmh.2005.06.005.

Yin, W., et al. 2013. Challenges and needs for China to eliminate rabies. Infect Dis Poverty, 2, 23.DOI:10.1186/2049-9957-2-23. 